\title{
Shape familiarity modulates preference for curvature in drawings of common-use objects
}

\author{
Erick G. Chuquichambi ${ }^{\text {Corresp., } 1}{ }^{,}$Letizia Palumbo ${ }^{2}$, Carlos Rey $^{1}$, Enric Munar $^{1}$ \\ ${ }^{1}$ Human Evolution and Cognition Group (EvoCog), University of the Balearic Islands, Palma, Balearic Islands, Spain \\ 2 Department of Psychology, Liverpool Hope University, Liverpool, United Kingdom \\ Corresponding Author: Erick G. Chuquichambi \\ Email address: erik.chuquichambi@uib.es
}

Drawing is a way to represent common-use objects. The contour of an object is a salient feature that defines its identity. Preference for a contour (curved or angular) may depend on how familiar the resulting shape looks for that given object. In this research, we examined the influence of shape familiarity on preference for curved or sharp-angled drawings of common-use objects. We also examined the possibility that some individual differences modulated this preference. Preference for curvature was assessed with a liking rating task (Experiment 1 ) and with a two-alternative forced-choice task simulating approach/avoidance responses (Experiment 2). Shape familiarity was assessed with a familiarity selection task where participants selected the most familiar shape between the curved and the angular version for each object, or whether both shapes were equally familiar for the object. We found a consistent preference for curvature in both experiments. This preference increased when the objects with a curved shape were selected as the most familiar ones. We also found preference for curvature when participants selected the shape of objects as equally familiar. However, there was no preference for curvature or preference for angularity when participants selected the sharpangled shapes as the most familiar ones. In Experiment 2, holistic and affective types of intuition predicted higher preference for curvature. Conversely, participants with higher scores in the unconventionality facet showed less preference for the curved drawings. We conclude that shape familiarity and individual characteristics modulate preference for curvature. 


\section{Shape familiarity modulates preference for curvature} 3 in drawings of common-use objects

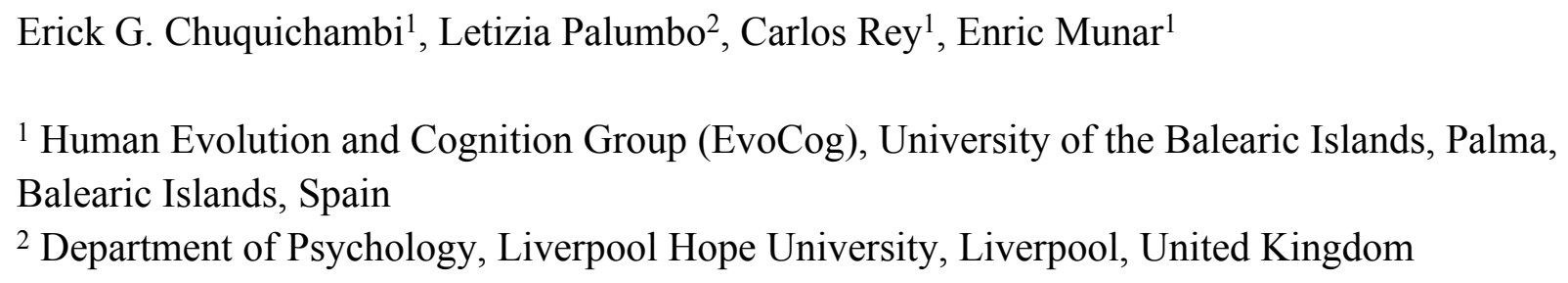

\section{Abstract}

Drawing is a way to represent common-use objects. The contour of an object is a salient feature that defines its identity. Preference for a contour (curved or angular) may depend on how familiar the resulting shape looks for that given object. In this research, we examined the influence of shape familiarity on preference for curved or sharp-angled drawings of common-use objects. We also examined the possibility that some individual differences modulated this preference. Preference for curvature was assessed with a liking rating task (Experiment 1) and with a two-alternative forced-choice task simulating approach/avoidance responses (Experiment 2). Shape familiarity was assessed with a familiarity selection task where participants selected the most familiar shape between the curved and the angular version for each object, or whether both shapes were equally familiar for the object. We found a consistent preference for curvature in both experiments. This preference increased when the objects with a curved shape were selected as the most familiar ones. We also found preference for curvature when participants selected the shape of objects as equally familiar. However, there was no preference for curvature or preference for angularity when participants selected the sharp-angled shapes as the most familiar ones. In Experiment 2, holistic and affective types of intuition predicted higher preference for curvature. Conversely, participants with higher scores in the unconventionality facet showed less preference for the curved drawings. We conclude that shape familiarity and individual characteristics modulate preference for curvature.

\section{Introduction}

Common-use objects are perceived as utilitarian, familiar and hedonic products (Wang, $\mathrm{Yu} \& \mathrm{Li}$, 2019). These characteristics influence how we interact with them daily. For instance, utility, 
40 familiarity and/or hedonism might be factors that contribute to generally preferring common-use 41 objects with curved contours over sharp-angled ones (Bar \& Neta, 2006, 2007; Munar et al., 42 2015). Preference for curvature was shown using drawings of car interiors (Leder \& Carbon,

43 2005), pictures of windows (Naghibi Rad et al., 2019), furniture (Dazkir \& Read, 2012), product 44 packaging (Westerman et al., 2012), exterior façades (Ruta et al., 2019) and interior architectural 45 environments (Van Oel \& Van den Berkhof, 2013; Vartanian et al., 2013, 2017), among others. 46 While most of these stimuli involve representational content, preference for curvature was also 47 found using non-representational art-related stimuli such as abstract artworks (Ruta et al., 2021) 48 or abstract shapes and patterns (Bertamini et al., 2016, 2019).

49 Previous studies suggested that shared preferences are more usual with representational stimuli

50

51

52

53

54

55

56

57

58

59

60

61

62

63

64

65

66

67

68

69

70

71

72

73

74

75

76

77

78 than abstract stimuli (Vessel \& Rubin, 2010; Schepman et al., 2015; Schepman, Rodway \& Pullen, 2015). Rodway et al. (2016) proposed that liking for representational stimuli is influenced by associations developed with the subject matter or semantic content of the picture. Therefore, our experience with the representational content of drawings or with the way an object is represented might also make preference for these stimuli more systematic and predictable. Skilled artists design representational drawings with relative ease (Kozbelt et al., 2010). On the one hand, the design process involves decisions about proportions, shading, lines, or colors, among others. On the other hand, the design process also involves implicit constraints such as the objects' functionality and usability, and sometimes even the cost of production (Lawson, 1980; Kavakli et al., 1999; Bertamini \& Sinico, 2019).

\section{Preference for curvature and familiarity}

The consistency of visual preference for the representational content of stimuli highlights its association with familiarity (Reber, Winkielman \& Schwarz, 1998; Reber, Wurtz \& Zimmermann, 2004). Berlyne (1971) considered that familiarity strongly influences the psychobiological mechanisms underlying aesthetic experiences. Therefore, increased exposure to specific visual features might also modulate the potential preference for the same visual features. In this regard, some studies suggested that curved contours are more frequent in natural scenes than sharp-angled ones (Koenderink, 1984; Hoffman \& Singh, 1997). Ruta et al. (2019) used a dynamic computational model of the visual cortex and a model that characterizes discomfort in terms of adherence to the statistics of natural images (Penacchio, Otazu \& Dempere-Marco, 2013; Penacchio \& Wilkins, 2015) to analyze the statistical properties of drawings of architectural façades with different contour types (curved, mixed, sharp-angled and rectilinear). They found that stimulus preference was related in both models and it matched the behavioural findings of preference for façades. Therefore, they suggested that the link between the statistical properties of natural scenes and preference for curvature might have evolved from human interaction with natural environments. Other studies suggested a faster speed of processing smooth contours over angular ones (Bertamini, Palumbo \& Redies, 2019; Chuquichambi et al., 2020). Bertamini, Palumbo and Redies (2019) argued that this advantage may be explained because curved features tend to match the statistics of the natural environment in which the 
79 visual system has evolved. However, preference for curvature may also be a context-specific

80 effect, and not extend to all natural environment stimuli (Hůla \& Flegr, 2016).

81 The influence of familiarity on preference might be also related to the proximity of an object to

82 the category prototype. In general, we would expect a link between typicality and familiarity

83 (Hekkert, Snelders \& Wieringen, 2003). Whitfield and Slatter (1979) investigated whether

84 proximity to the prototype influenced aesthetic choice using images of furniture with different

85 styles. These authors found that the furniture and styles selected by participants in their similarity

86 task consistently corresponded to those selected in their aesthetic task. Influenced by these

87 results, Whitfield and Slatter (1979) developed the preference for prototypes theory suggesting

88 that aesthetic choice reflects categorization and prototypicality. That is, prototypicality may act

89 as an influential determinant of preference for everyday objects (Whitfield, 1983). Winkielman

90 et al. (2006) proposed that part of preference for prototypicality arises from a general mechanism

91 linking fluency and positive affect. Along with prototypicality, these authors suggested that other

92 factors might also act as fluency-enhancing variables and, therefore, explain the prototypicality-

93 attractiveness relationship. In this sense, preference for objects with curved contours might be

94 one of these variables because curvature facilitates processing fluency (Corradi \& Munar, 2020).

95 Drawings of common-use objects are characterised by meaningful and familiar content (Hekkert

96 \& Snelders, 1995; Hekkert, Snelders \& Wieringen, 2003). They involve the perceiver's previous

97 knowledge and momentary perceptual experience (Leder et al., 2004). Given that people might

98 be more exposed to curved contours than to sharp-angled ones in daily life, the potential

99 preference for curved drawings of common-use objects might be modulated or explained by the

100 degree of familiarity of these objects. However, this relationship might be also modulated by the

101 artistic reproduction of drawings.

\section{Drawings as artistic works}

103 Drawings are associated with innovation and creativity because of their art-related nature

104 (Purcell \& Gero, 1998). The experience of drawing embodies abstract and high-level design

105 ideas, and allows some degree of uncertainty about how to represent the physical attributes of the

106 object (Gross et al., 1988). These characteristics might differentiate preference for

107 representational drawings from preference for more realistic (e.g., photographs) or more abstract

108 stimuli (e.g., irregular polygons). Contrary to representational stimuli, Bornstein (1989) found

109 that abstract paintings, drawings, and matrices did not show a strong mere exposure effect. This

110 effect proposes that affect increases with repeated unreinforced exposure of a stimulus, and

111 therefore, familiarity (Zajonc, 1968). Leder (2001) also showed that repeated exposure had little

112 effect on art-related stimuli. Instead, he suggested that familiarity-liking relations were weakened

113 by knowledge and were greater in spontaneous judgements. These findings are compatible with

114 the fact that novelty is an important factor in the appreciation of fine arts, where the seeking for

115 novelty is a dominant force in its development (Matindale, 1990). Hekkert, Snelders and

116 Wieringen (2003) showed typicality and novelty as equally effective predictors to explain

117 aesthetic preference of consumer products (e.g., telephones, cars, etc.). They suggested that there

118 should be a balance between novelty and typicality in the design of common-use objects. 
119 Interestingly, Park, Shimojo and Shimojo (2010) found segregation of preference across objects' 120 categories, with familiarity dominant in faces, and novelty dominant in natural scenes. Given this 121 context, the interaction between the representational content and art-related characteristics of 122 drawings of common-use objects might contribute to understanding the role of familiarity in 123 predicting aesthetic judgements (Sluckin, Hargreaves \& Colman, 1982).

\section{Individual differences and preference for curvature}

125 Individual differences also modulate aesthetic judgements (Child, 1962, 1965; Leder et al., 126 2019). However, the influence of individual differences in preference for curvature diverges 127 between studies. Silvia and Barona (2009) investigated the role of artistic expertise in preference 128 for curvature using arrays of circles and hexagons, and asymmetrical random polygons. Although they found an interaction between art training with angular stimuli, this interaction changed depending on the specific stimuli set. Vartanian et al. (2017) also found divergent results in preference for curvature among experts (architects or designers) and non-experts. They presented these participants with images of curvilinear and rectilinear architectural interior spaces in a beauty judgement task and an approach-avoidance decision task. Despite that the experts found curvilinear spaces more beautiful than rectilinear ones, contour did not affect their willingness to enter or exit these spaces. Conversely, contour had no effect on judgements of beauty among nonexperts, but they were more likely to enter curvilinear spaces than rectilinear ones. However, a more recent study did not confirm preference for curved interior spaces with quasi-experts in industrial design (Palumbo et al., 2020), hence highlighting that individual differences might also depend on the specific training received in the area of expertise. Cotter et al. (2017) also reported that artistic expertise, a personality trait such as openness to experience, along with other cognitive traits (i.e., holistic thinking) predicted higher preference for curvature using irregular polygons, but not using arrays of circles and hexagons. Corradi et al. (2019a) suggested that aesthetic sensitivity to curvature coexists with a remarkable individual variation on people's judgements. They presented real objects and abstract designs to art and non-art students in a two-alternative forced-choice task. They also were interested in the role of sex, openness to experience and artistic expertise. Both groups of students preferred the curved stimuli but none of the individual variables showed significant results. The present study In this study, we examined preference for contour (curved or angular) in two experiments using drawings of common-use objects. The drawings consisted of pairs of the same object with a curved and a sharp-angled version created by quasi-expert students in Design as described in Bertamini and Sinico (2019). They were rated by non-experts for seven characteristics, confirming an association between curvature and beauty. In the current experiments, we examined whether the selection of pairs based on the familiarity of the shape of the objects, and specific individual differences, would modulate preference for contour. Each experiment had two tasks. The first tasks were a liking rating task for the drawings in Experiment 1, and a twoalternative forced-choice (2AFC) task simulating approach/avoidance responses in Experiment 2. 
159

160

161

162

163

164

165

166

167

168

169

170

171

172

173

174

175

176

177

178

179

180

181

182

183

184

185

186

187

188

189

190

191

192

193

194

195

196

197

198

experiments. In this task, participants categorized the object pairs in three groups: (a) the pairs in which the curved shape was the most familiar, (b) the pairs in which the sharp-angled shape was the most familiar, and (c) the pairs in which both shapes were equally familiar. This way, we could analyse preference for curvature in each group. At the end of the experimental tasks, all participants were administered a set of individual measures: a Spanish adapted scale of Art interest and Art knowledge (Chatterjee et al., 2010), the Openness to experience Scale from the NEO-FFI (McCrae \& Costa, 2004), the items of the Unconventionality facet from the HEXACO personality test (Lee \& Ashton, 2004), and the Types of Intuition Scale (TIntS) (Pretz et al., 2014).

First, we hypothesized that participants would prefer the curved object drawings in both experiments because preference for curvature has shown to be consistent across different stimuli and experimental tasks (Palumbo \& Bertamini, 2016; Chuquichambi et al., 2021). Second, we expected that the curved contours would be perceived as the most familiar because of the predominant role of curvature on shape's perception (Pasupathy \& Connor, 2002) and its suggested higher exposure in nature (Koenderink, 1984; Hoffman \& Singh, 1997; Bertamini, Palumbo \& Redies, 2019; Ruta et al., 2019). Third, familiarity selection for curved shapes might largely explain preference for curved drawings or only influence this preference. That is, we could find that when the curved shapes are selected as the most familiar, the higher the preference for the curved drawings, or we could find preference for the curved drawings without necessarily perceiving the curved shapes as the most familiar. Fourth, according to the divergences between studies, the variation in people's judgements and stimulus characteristics might explain the inconsistent role of some individual differences in preference for curvature (Corradi et al., 2019b). Therefore, the current study aimed to assess to what extent preference for curvature might be explained by familiarity for the shape with which the objects were represented in the drawings and whether this would be modelled by individual differences.

\section{Experiment 1}

\section{Materials \& Methods}

\section{Participants}

Forty-nine adult students (41 female, Mage $=21.3$, SDage $=4.95$ ) at the University of the Balearic Islands (UIB) volunteered to participate in the experiment. All participants reported normal or corrected to normal vision and were naïve concerning the experimental hypothesis. They provided written informed consent before the experiment. The experiment was conducted following the code of practice of the APA guidelines, and received ethical approval from the Committee for Ethics in Research (CER) of the UIB (Ref: IB 3828/19 PI).

\section{Apparatus and materials}

Ninety drawings of familiar objects were selected from the IUAV image database (https://osf.io/cx62j/) (Bertamini \& Sinico, 2019). The selected stimuli consisted of 45 pairs of drawings. Each pair represented the same object, a curved and a sharp-angled version. These pairs were selected considering that the curved and the sharp-angled versions were similar in terms of size, compression ratio of the file (an index used as a measure of image complexity; 
199 Forsythe, Mulhern \& Sawey, 2008; Palumbo et al., 2014), perceived lightness, weight, or style

200

201

202

203

204

205

206

207

208

209

210

211

212

213

214

215

216

217

218

219

220

221

222

223

224

225

226

227

228

229

230

231

232

233

234

235

236

237

238

according to the data reported by Bertamini and Sinico (2019). On the other hand, some pairs of drawings differed in how they were made. Thirty pairs were hand-made and 15 were computermade. Similarly, 15 pairs were shaded and 30 were not shaded. Lastly, the apparent position of the objects in relation to the viewer corresponded to a frontal view in 24 pairs, and to $a 3 / 4$ view in 21 pairs. Out of the hand-made drawings, 13 pairs were shaded, 17 pairs were not shaded, 13 pairs were in $3 / 4$ view, and 17 pairs were in frontal view. Out of the computer-made drawings, 2 pairs were shaded, 13 pairs were not shaded, 8 pairs were in $3 / 4$ view, and 7 pairs were in frontal view. The curved and the sharp-angled version of each pair had the same Category, Shading and Position. The pairs of stimuli were equalized in size and had 300 dpi resolution. Every stimulus was presented framed on an outline of 600 pixels height, and 600 pixels width. (Fig. 1).

Please insert Figure 1 about here

We used the same drawings in the liking rating task and the familiarity selection task. The liking task recorded ratings of each drawing using a horizontal sliding bar from 0 to 100 . The ends of the bar had the labels "I don't like it" (0) on one side, and "I like it very much" (100) on the other side (Fig. 2A). Each stimulus was presented on the centre of the screen until the participant had responded on the sliding bar using the mouse. The task had 8 practice trials corresponding to 4 additional pairs of drawings from the image database, and 90 experimental trials corresponding to the 45 stimuli pairs. Trial sequence was randomized.

The familiarity selection task presented each pair of drawings simultaneously, one on the left and the other on the right side of the screen, until the participant responded. The question was "Which shape is the most familiar for this object?" There were three-alternative responses labelled as left, equal, and right. If they chose the shape of the left-side object as the most familiar, they had to press the left key. If they chose the shape of the right-side object, they had to press the right key. They could also choose the shape of both objects as equally familiar by pressing the central key. The task had 8 practice trials and 45 experimental trials corresponding to the 45 pairs. Left-side and right-side presentation and trial sequence were randomized.

Please insert Figure 2 about here

Four questionnaires were administered. The first was an Art interest and Art knowledge scale adapted from Chatterjee et al.'s (2010) Art Training, Interest and Activities Scale. This scale was used in previous studies of aesthetic sensitivity (e.g., Corradi et al., 2019b). It consists of eight items with a 0-6 Likert scale. Five items (1-5) measure interest in art, and three (6-8) measure formal education in art. The second questionnaire was the Openness to experience Scale of the NEO-FFI (McCrae \& Costa, 2004). It consists of twelve items rated on a scale ranging from 1 (strongly disagree) to 5 (strongly agree). The third questionnaire consists of four items about the Unconventionality facet of the Openness to experience domain from the HEXACO 100

Peer) reviewing PDF | (2021:01:57478:2:0:NEW 15 Jun 2021) 
239 Personality Inventory-Revised (Lee \& Ashton, 2004). We included this measure because Cotter

240 et al. (2017) showed that higher scores on the Unconventionality facet predicted greater

241 preference for curvature using geometrical patterns. Finally, participants completed the Types of

242 Intuition Scale (TIntS) to examine whether the way people make decisions and solve problems

243 modulates preference for drawings (Pretz et al., 2014). This scale consists of 23 items (e.g., "I

244 am a 'big picture' person', "I tend to use my heart as a guide for my actions") rated on a scale

245 ranging from 1 (definitely false) to 5 (definitely true). The items are grouped into four subscales:

246 Holistic Abstract (HA, thinking about a problem in abstract terms), Holistic Big Picture (HB,

247 focusing on the entire problem rather than details of the situation), Inferential (I, making

248 decisions based on automatic, analytic processes), and Affective (A, making decisions by relying

249 on emotional reactions to a situation). The scores of the questionnaires are reported in Table 1.

250

251

252

253

254

255

256

257

258

259

260

261

262

263

264

265

266

267

268

269

270

271

272

273

274

275

276

277

278

Please insert Table 1 about here

All tasks were designed with OpenSesame (3.2) software (Mathôt, Schreij \& Theeuwes, 2012). They were implemented in computers equipped with Intel i5 processors and 21-inch screens set at $1920 \times 1080$ pixels.

\section{Procedure}

The experimental session was carried out at the Psychology Laboratory of the UIB, using isolated cabins and individual computers with the same software and light conditions.

Participants were welcomed at the laboratory and they provided written informed consent. They received verbal and written instructions before starting each task. The liking task was the first one. Participants were told that a drawing would be presented at the centre of the computer screen. They had to indicate how much they liked the drawing with a mouse click on the horizontal sliding bar. Next, participants carried out the familiarity selection task. They were told that pairs of drawings would be presented on the computer screen, one on the left and the other on the right side of the screen. They had to select which shape was the most familiar for the object in the drawing, or whether both shapes were equally familiar, by pressing the appropriate key. After these tasks, participants filled in the four questionnaires. The experimental session lasted about 20 minutes. Finally, participants were debriefed and thanked.

\section{Data analysis}

Data analysis was carried out with the $\mathrm{R}$ environment for statistical computing (R Core Team, 2018). Participants' responses in the liking task, the familiarity selection task and questionnaires were analysed by means of linear mixed effects models (Hox, 2010; Snijders \& Bosker, 2012). These models account simultaneously for the between-subject and within-subject effects of the independent variables (Baayen, Davidson \& Bates, 2008). They have been previously used to analyse preference judgements and individual differences (e.g., Corradi et al., 2019a; 2019b). The 'Imer' function from the lme4 package was used to fit the models (Bates et al., 2015). The afex package (Singmann et al., 2016), with the likelihood ratio test, was used to produce the inferential statistics and $\mathrm{p}$ values. The lsmeans package was used to obtain predicted means for 
279 the fixed effects (Lenth, 2016). Participant and Stimulus were included as random effects in all

280 models. Model selection was carried out considering model fit indices and following Barr et al.'s 281 (2013) and Brauer and Curtin's (2018) guidelines to choose the maximal random-effects 282 structure justified by the experimental design. Finally, we performed a study of influential cases 283 based on Cook's distance (Cook's D) in each model. This measure evaluates each participant's 284 influence on the results by examining the impact of its removal from the data set (Corradi et al., 285 2018).

\section{Results}

287 We considered three models. The first model tested preference for curvature and its relation to 288 the other stimulus properties: computer-made versus hand-made, shaded versus not shaded, and

289

290

291

292

293

294

295

296

297

298

299

300

301

302

303

304

305

306

307

308

309

310

311

312

313

314

315

316

317

318

frontal versus $3 / 4$ view. The second model analysed the relationship between preference for curvature and familiarity selection. The third model tested the influence of the individual measures (i.e., personality and art expertise) on the liking ratings related to preference for curvature.

The first model aimed to predict liking ratings based on Contour (curved vs. sharp-angled), Category (computer-made vs. hand-made), Shading (shaded vs. not shaded), and Position (frontal vs. $3 / 4$ view) as factors of fixed effects. We also included the interactions between Contour and Category, Contour and Shading, and Contour and Position. The best model, according to models fit indices, included random slopes within participant random effect. Influential cases analysis revealed no influential cases whose value exceeded the recommended cut-off point, which was .090. Participants significantly liked the curved drawings ( $\mathrm{M}=55.1$, $95 \% \mathrm{CI}[50,60.2])$ more than the sharp-angled ones $(\mathrm{M}=50.4,95 \% \mathrm{CI}[45.4,55.4]), \beta=3.51$, $\mathrm{SE}=1.5, \mathrm{t}(92.8)=2.31, p=.023,95 \% \mathrm{CI}[.53,6.5]($ Fig. 3A). There was no significant interaction of Contour $x$ Category, $\beta=-1.94, \mathrm{SE}=1.52, \mathrm{t}(4217)=-1.28, p=.20,95 \% \mathrm{CI}[-4.9,-$ 1.04], Contour $x$ Shading, $\beta=1.85, \mathrm{SE}=1.6, \mathrm{t}(364)=1.16, p=.24,95 \% \mathrm{CI}[-1.3,5]$, or Contour $\mathrm{x}$ Position, $\beta=-2.45, \mathrm{SE}=1.4, \mathrm{t}(4217)=-1.76, p=.080,95 \% \mathrm{CI}[-5.2, .30]$. Participants also significantly liked the drawings with shading $(\mathrm{M}=61.7,95 \% \mathrm{CI}[54,69.4])$ more than the drawings with no shading $(\mathrm{M}=43.8,95 \% \mathrm{CI}[38.3,49.3]), \beta=17, \mathrm{SE}=4.64, \mathrm{t}(64)=3.66, p<$ $.001,95 \% \mathrm{CI}[7.9,26.1]$. There was no significant difference between the hand-made $(\mathrm{M}=50.6$, $95 \% \mathrm{CI}[45.5,55.6])$ and the computer-made drawings, $(\mathrm{M}=55,95 \% \mathrm{CI}[47.6,62.3]), \beta=-3.4$, $\mathrm{SE}=4.1, \mathrm{t}(44)=-.83, p=.41,95 \% \mathrm{CI}[-4.6,11.4]$. Similarly, liking ratings did not significantly differ between the drawings in frontal $(\mathrm{M}=50.7,95 \% \mathrm{CI}[44.5,57])$ and $3 / 4$ view $(\mathrm{M}=54.8$, $95 \% \mathrm{CI}[48.8,60.7]), \beta=-2.82, \mathrm{SE}=3.7, \mathrm{t}(44)=-.75, p=.45,95 \% \mathrm{CI}[-10.1,4.5]$.

The familiarity selection task showed that the curved shapes were selected as the most familiar ones in a proportion of .49, the sharp-angled shapes were selected as the most familiar ones in a proportion of .22, and both shapes were selected as equally familiar in a proportion of .29. The second model included liking rating as the variable to be predicted, and Contour type and Familiarity as categorical fixed effects. The interaction between the two factors was also included, as our main objective was to examine the relationship between contour preference and familiarity. The three familiarity categories were included in the analysis as three levels: the

Peer) reviewing PDF | (2021:01:57478:2:0:NEW 15 Jun 2021) 
319

320

321

322

323

324

325

326

327

328

329

330

331

332

333

334

335

336

337

338

339

340

341

342

343

344

345

346

347

348

349

350

351

352

353

354

355

356

357

358

curved shape selected as the most familiar, the sharp-angled shape selected as the most familiar, and both shapes selected as equally familiar. The best model included random slopes within participant and stimulus. Influential cases analysis revealed two influential cases exceeding the recommended cut-off point, which was .087 . Therefore, these participants were excluded from the analysis. Results showed that the Contour $x$ Familiarity interaction was significant when we considered the curved and sharp-angled responses in the Familiarity factor, $\beta=12.58, \mathrm{SE}=2.22$, $\mathrm{t}(82.5)=5.67, p<.001,95 \%$ CI $[8.23,16.93]$. Specifically, participants liked the curved drawings $(\mathrm{M}=52.9,95 \% \mathrm{CI}[47.3,58.4])$ more than the sharp-angled ones $(\mathrm{M}=43.3,95 \% \mathrm{CI}$ $[38,48.7])$ when the curved shapes were selected as the most familiar ones, $\beta=9.6, \mathrm{SE}=1.6$, $\mathrm{t}(44)=5.8, p<.001$. Conversely, when the sharp-angled shapes were selected as the most familiar ones, liking ratings did not differ significantly between the sharp-angled drawings $(\mathrm{M}=$ $50.3,95 \% \mathrm{CI}[43.7,56.8])$ and the curved ones $(\mathrm{M}=47.3,95 \% \mathrm{CI}[41.8,52.8]), \beta=3, \mathrm{SE}=2.1$, $\mathrm{t}(23.7)=1.4, p=.16$ (Fig. 3B). The Contour $\mathrm{x}$ Familiarity interaction was significant when we considered both shapes selected as equally familiar and the sharp-angled shapes selected as the most familiar ones, $\beta=8.46, \mathrm{SE}=2.2, \mathrm{t}(79)=3.79, p<.001,95 \% \mathrm{CI}[4.1,12.8]$. This effect revealed that when both the curved and sharp-angled shapes were selected as equally familiar, participants still liked the curved drawings $(\mathrm{M}=50.8,95 \% \mathrm{CI}[45,56.6])$ more than the sharpangled ones $(\mathrm{M}=45.2,95 \% \mathrm{CI}[39.5,50.8]), \beta=5.6, \mathrm{SE}=1.5, \mathrm{t}(25)=3.6, p=.0010$. Lastly, the Contour $x$ Familiarity interaction also reached significance when we considered the curved shapes selected as the most familiar ones and both shapes selected as equally familiar, $\beta=4.01$, $\mathrm{SE}=1.87, \mathrm{t}(102.1)=2.15, p=.034,95 \% \mathrm{CI}[.36,7.67]$. In conclusion, we found an effect of preference for curvature when the curved shapes were selected as the most familiar ones and when both shapes were selected as equally familiar. However, there was no effect of preference for contour when the sharp-angled shapes were selected as the most familiar ones.

Please insert Figure 3 about here

Regarding the individual measures, we analysed whether they modulated liking ratings related to the curved and sharp-angled drawings. The model predicted liking ratings based on Contour and its interactions with Art interest, Art knowledge, Openness to experience, the Unconventionality facet, and TIntS subscales (HA, HB, I and A) as predictors. All continuous predictors were centred on the grand mean. The best model included random slopes within participant and stimulus. Influential cases analysis showed no influential cases whose value exceeded the recommended cut-off point, which was .10. Results revealed that participants who scored higher in the Holistic Big Picture Subscale (HB) showed higher liking ratings for all the drawings, $\beta=$ $1.3, \mathrm{SE}=.52, \mathrm{t}(23)=2.5, p=.020,95 \% \mathrm{CI}[.28,2.32]$. All other effects and interactions were nonsignificant. All effects are included in Table S1 as supplementary material.

\section{Discussion}

Experiment 1 showed that participants liked the curved drawings more than the sharp-angled ones. This result supports the curvature effect (Corradi \& Munar, 2020). Our results also 
359

360

361

362

363

364

365

366

367

368

369

370

371

372

373

374

375

376

377

378

379

380

381

382

383

384

385

386

387

388

389

390

391

392

393

394

395

396

397

398

reported an interaction between familiarity and curvature on shape preference. When the curved shapes were selected as the most familiar ones, the curved drawings were liked more than the sharp-angled drawings. This finding supports the role of familiarity in predicting aesthetic preference (Verhaeghen, 2018; Chmiel \& Schubert, 2019). That is, the drawings with the shapes that were chosen as most familiar to represent the objects were liked more. However, we also found that when the shapes of the objects were selected as equally familiar, participants also liked the curved drawings more than the sharp-angled ones. Furthermore, when the sharp-angled shapes were selected as the most familiar ones, liking did not differ between the curved and sharp-angled drawings. Altogether, these findings suggest that familiarity of the shape with which the objects have been represented in the drawings modulates preference for curvature, but it does not completely explain participants' preference for the curved drawings. Individual measures analysis showed that participants with higher scores in the Holistic Big Picture subscale liked all the drawings more than participants with lower scores. All the other measures did not significantly influence liking ratings. These findings are in line with studies suggesting an uncertain role of some individual measures on preference for curvature (Corradi et al., 2019b).

\section{Experiment 2}

Experiment 2 consisted of a $2 \mathrm{AFC}$ task simulating approach/avoidance responses (Fig. 2B).

Approach/avoidance procedures have been previously used in preference for curvature research (Vartanian et al., 2013; Palumbo, Ruta \& Bertamini, 2015). Participants carried out the same familiarity selection task and questionnaires as in Experiment 1. In the 2AFC task, each pair of drawings was presented on the screen until participants responded, as in previous studies (Munar et al., 2015; Corradi et al., 2018). However, although these studies reported preference for images of curved real objects in short and medium presentation times, the effect disappeared in the until-response condition. Similarly, these authors reported preference for curved abstract patterns in short and medium presentation times, but in this case, the effect increased in the untilresponse condition. Palumbo and Bertamini (2016) showed that preference for curvature was consistent across tasks using irregular shapes. Considering these studies and the results from Experiment 1, we expected that participants would prefer the curved object drawings more than the sharp-angled ones. Furthermore, we expected that shape familiarity would also modulate preference for curvature.

\section{Materials \& Methods}

\section{Participants}

Forty-nine adult students ( 35 female, $\mathrm{M}_{\mathrm{age}}=26.3, \mathrm{SD}_{\mathrm{age}}=6.5$ ) at the UIB volunteered to participate in the experiment. All participants reported normal or corrected to normal vision and were naïve concerning the experimental hypothesis. They provided written informed consent before the experiment and were treated following the code of practice of the APA guidelines. The study received ethical approval from the Committee for Ethics in Research (CER) of the UIB (Ref: IB 3828/19 PI).

\section{Apparatus and materials}


399 We used the same 90 drawings as in Experiment 1 (Fig. 1). They were presented both in the 400 2AFC task and the familiarity selection task. In the 2AFC task, each pair of stimuli was 401 presented until response, a drawing on the left and the other on the right side of the computer 402 screen (Fig. 2B). Participants were instructed to select one of the two object drawings, and 403 instructions avoided the words 'liking', 'wanting' and 'preference' as in Munar et al. (2015) and 404 Corradi et al. (2018). Later, the selected drawing was enlarged to twice its previous size, while 405 the non-selected one was shrunk to half its previous size at the same position for $1000 \mathrm{~ms}$. This 406 action simulated an approach/avoidance behaviour (Bamford et al., 2015). As in Experiment 1, 407 the 2 AFC task had 8 practice trials with additional stimuli from the image database, and 45 408 experimental trials corresponding to the 45 pairs of drawings. Left-side and right-side stimulus presentation and trial sequence were randomized. The familiarity selection task and the set of questionnaires were the same as in Experiment 1. The scores of the questionnaires are reported in Table 2.

412

Please insert Table 2 about here

414

415

416

417

418

419

420

421

422

423

424

425

426

427

428

429

430

431

432

433

434

435

436

437

438

\section{Procedure}

The experimental session was carried out as in Experiment 1. Participants received verbal and written instructions before starting each task. First, they carried out the 2AFC task. They were told that they had to select one of two drawings presented on the screen using the right and left arrow keys. Then, the size of the selected drawing would be enlarged, and the size of the nonselected drawing would be shrunk. Next, they carried out the familiarity selection task receiving the same instruction as in Experiment 1. Lastly, they filled in the questionnaires using the same computer. The experimental session lasted about 20 minutes. Finally, participants were debriefed and thanked.

\section{Data analysis}

Analyses were carried out with the R environment for statistical computing (R Core Team, 2018). We mainly modelled responses by means of generalized linear mixed effects models given that the dependent variable in the 2AFC task was the kind of contour participants selected (curved or sharp-angled). The 'glmer' function from the lme4 package was used to fit the models (Bates et al., 2015). All models included Participant and Stimulus as random effects. Model selection was performed following the same considerations outlined in Experiment 1. Finally, we performed a study of influential cases in each model.

\section{Results}

We considered three analyses. First, we analysed preference for curvature and its relationship with the other stimulus characteristics. The second analysis was based on a model to test the relationship between preference for curvature and familiarity selection. The third analysis examined the influence of the individual measures on preference for curvature.

Previously, we carried out a t-test on the preference for curvature as compared to angularity to examine participants preference choices in the 2AFC. Results showed that participants chose the 
439 curved drawings significantly above chance level $(\mathrm{M}=.61), t(48)=5.54, p<.001,95 \%$ CI $[.57$,

$440.65], d=.79$ (Fig. 4A). Next, we modelled the curved choices as the variable to be predicted. The 441 model included Category (computer-made vs. hand-made), Shading (shaded vs. not shaded),

442 Position (frontal vs. $3 / 4$ view), and the interaction between these factors as fixed effects. The best

443 model included random intercepts within participant and stimulus. Influential cases analysis

444 revealed no influential values exceeding the recommended cut-off point, which was .089. Results

445 revealed no significant effect either for Category, $\beta=-1.38, \mathrm{SE}=.76, \mathrm{Z}=-1.81, p=.070,95 \%$

446 CI $[-2.9, .11]$, Shading, $\beta=.02, \mathrm{SE}=.53, \mathrm{Z}=.04, p=.96,95 \% \mathrm{CI}[-1.02,1.06]$, or Position, $\beta=$ $447-.74, \mathrm{SE}=.53, \mathrm{Z}=-1.4, p=.16,95 \% \mathrm{CI}[-1.8, .30]$. Moreover, there was no significant

448 interaction between Category x Shading, $\beta=.27, \mathrm{SE}=.94, \mathrm{Z}=.29, p=.77,95 \% \mathrm{CI}[-1.57,2.1]$, 449 Category $x$ Position, $\beta=.90, \mathrm{SE}=.71, \mathrm{Z}=1.26, p=.21,95 \% \mathrm{CI}[-.50,2.3]$, or Shading $\mathrm{x}$

450 Position, $\beta=.75, \mathrm{SE}=.72, \mathrm{Z}=1.04, p=.30,95 \% \mathrm{CI}[-.66,2.15]$. These results indicated that

451 the choice of the curved drawing does not depend on the category of the drawing, whether or not 452 it is shaded, and whether it is in frontal or $3 / 4$ view.

453 On the other hand, the familiarity selection task showed that the curved shapes were selected as 454 the most familiar in a proportion of .45, the sharp-angled shapes were selected as the most 455 familiar in a proportion of .21, and both shapes were selected as equally familiar in a proportion of .34. We modelled whether familiarity selection predicted preference in the 2AFC task. The model included curved choices as the variable to be predicted. Familiarity (curved, equally,

459

460

461

462

463

464

465

466

467

468

469

470

471

472

473

474

475

476

477

478 sharp-angled) and Lateralization (left vs. right) were included as categorical fixed effects. The best model included random slopes within participant. Influential cases analysis revealed no extreme values exceeding the recommended cut-off point, which was .087. Results showed a main effect when we compared the pairs in which the curved shape was the most familiar and the pairs in which the sharp-angled shape was the most familiar, $\beta=1.90, \mathrm{SE}=.35, \mathrm{Z}=5.5, p<$ $.001,95 \%$ CI $[1.22,2.6]$. Post-hoc tests revealed that curved preference was higher when the curved shapes were selected as the most familiar $(\mathrm{M}=.83,95 \% \mathrm{CI}[.74, .89])$ than when the sharp-angled shapes were selected as the most familiar $(\mathrm{M}=.42,95 \% \mathrm{CI}[.31, .53])$, OR (Odds Ratio $)=6.72,95 \%$ CI $[4.1,13.2]$. That is, when participants selected the curved shapes as the most familiar ones, they also mostly preferred the curved drawings over the sharp-angled ones in the 2AFC task, but this was not the case when participants selected the sharp-angled shapes as the most familiar ones. Similarly, there was a main effect when we considered the curved shapes selected as the most familiar ones and both shapes selected as equally familiar, $\beta=1.22$, SE $=$ $.25, \mathrm{Z}=4.96, p<.001,95 \% \mathrm{CI}[.74,1.71]$. Curved preference choices were higher when the curved shapes were selected as the most familiar ones than when both shapes were selected as equally familiar $(\mathrm{M}=.59,95 \% \mathrm{CI}[.50, .67]), \mathrm{OR}=3.4,95 \% \mathrm{CI}[2.1,5.5]$. Lastly, there was a main effect when we considered both shapes selected as equally familiar and the sharp-angled shapes selected as the most familiar ones, $\beta=.68, \mathrm{SE}=.23, \mathrm{Z}=2.9, p=.0035,95 \% \mathrm{CI}[.22$, 1.14]. Post-hoc comparisons showed that curved preference was higher when participants selected both shapes as equally familiar than when they selected the sharp-angled shapes as the most familiar ones, $\mathrm{OR}=1.98,95 \% \mathrm{CI}[1.25,3.12]$ (Fig. 4B). These results suggest that 
479 participants preferred the drawings they chose as more familiar. They also support the findings

480 from Experiment 1, suggesting that shape familiarity modulates preference for curvature between 481 tasks.

482

483

484

485

486

487

488

489

490

491

492

493

494

495

496

497

Regarding the individual measures, we modelled whether they modulated contour preference choices. The model included Art interest, Art knowledge, Openness to experience, Unconventionality facet, and TIntS subscales (HA, HB, I and A) as predictors. These predictors were centred on the grand mean. The best model included random slopes within participant and stimulus. Influential cases analysis revealed four influential values exceeding the recommended cut-off point, which was .10. Thus, these participants were excluded from the analysis. Results showed that participants who scored higher in the HB subscale showed a significantly higher preference for curved drawings, $\beta=.13, \mathrm{SE}=.04, \mathrm{Z}=3.5, p<.001,95 \% \mathrm{CI}[.06, .20]$. Those who scored higher in the A subscale also showed a significantly higher preference for curved drawings, $\beta=.052, \mathrm{SE}=.016, \mathrm{Z}=3.3, p<.001,95 \% \mathrm{CI}[.02, .08]$. In contrast, participants who scored higher in the Unconventionality facet showed significant lower preference for curved drawings, $\beta=-.09, \mathrm{SE}=.04, \mathrm{Z}=-2.2, p=.028,95 \% \mathrm{CI}[-.17,-.01]$. The other effects were nonsignificant. All effects are included in Table $S 2$ as supplementary material.

\section{Correlations between experiments}

We analysed the correlation between the data from the two experiments to determine the consistency of responses to the same drawings from different participants. First, we performed a correlation analysis based on drawings between liking ratings in Experiment 1 and preference choices in Experiment 2. From Experiment 1, we calculated the difference between the liking for the curved drawing and the sharp-angled drawing of each pair. We correlated these values with the choice mean (between 0 a 1) for each pair of drawings from the 2AFC task in Experiment 2. Subsequently, the bias-corrected and accelerated CI was calculated using the bootstrap resampling method with 1499 samples suggested for a test at the 0.05 and 0.01 level (Davidson $\&$ MacKinnon, 2000). Results revealed a significant positive correlation between the liking ratings and curved preference choices, $r_{\mathrm{s}}(45)=.66, p<.001,95 \% \mathrm{CI}[.46, .80]$. This result supported a positive relationship of preference for drawings between tasks (Fig. 5A). Second, we compared the familiarity responses of participants in the two experiments. We obtained a familiarity value for each pair of stimuli regarding the three-alternative responses from the familiarity selection tasks. That is, we grouped the trials where participants selected the curved shape as the most familiar (+1), the sharp-angled shape as the most familiar (-1) and both

515 stimuli. Then, we correlated these values between both familiarity selection tasks and calculated

$516 \mathrm{CI}$ as in the first correlation. Results showed a strong positive association of familiarity

517 judgements between the two experiments, $r_{s}(45)=.92, p<.001,95 \%$ CI $[.88, .94]$. These results 
518 supported that familiarity with the shape of the objects was consistent across different

519 participants (Fig. 5B).

520

521

522

523

524

525

526

527

528

529

530

531

532

533

534

535

536

537

538

539

540

541

542

543

544

545

546

547

548

549

550

551

552

553

554

555

556

557
Please insert Figure 5 about here

\section{Discussion}

Experiment 2 showed that participants preferred the curved drawings over the sharp-angled ones. This result supported our main hypothesis about the curvature effect (Corradi \& Munar, 2020). Therefore, together with the results from Experiment 1, we suggest a consistent preference for the curved drawings of common-use objects between tasks.

We also found that familiarity for the curved stimuli predicted a higher preference for curvature in the 2AFC task than familiarity for the sharp-angled stimuli and the stimuli selected as equally familiar. That is, when the curved shapes were selected as the most familiar ones, there was a higher preference for curvature. Similarly, there was preference for curvature when participants selected both shapes as equally familiar. In contrast, we did not find preference for angularity or for curvature when participants selected the sharp-angled shapes as the most familiar ones. These results support the influence of familiarity on preference. However, they also showed that familiarity is not the only factor determining preference for drawings of common-use objects. On the other hand, some individual measures influenced preference choices. Specifically, participants who scored higher in the HB and A subscales of the TIntS showed a higher preference for the curved drawings. In contrast, those who scored higher in the Unconventionality facet showed less preference for the curved drawings. These results suggest that the $2 \mathrm{AFC}$ task is a more sensitive procedure to find the potential influence of individual differences in preference for curvature than the liking rating task. Conversely, the results also suggest an uncertain influence of some individual measures (e.g., art expertise or openness to experience) on preference for curvature (Corradi et al., 2019b).

Finally, the correlation analysis between the data from the two experiments showed a similar pattern of preference for the pair of drawings. On the other hand, the perception of familiarity with the shape of the objects and their representational content was highly consistent using two different groups of participants.

\section{General discussion}

We examined preference for curvature and its relationship with familiarity using drawings of common-use objects in two experiments. Experiment 1 consisted of a liking rating task, a familiarity selection task, and a set of individual measures. Experiment 2 used the same stimuli and different participants, and consisted of a $2 \mathrm{AFC}$ task simulating approach/avoidance responses, and the same familiarity selection task and individual measures of Experiment 1. In Experiment 1, we found higher liking ratings for the curved than the sharp-angled drawings. Similarly, in Experiment 2, participants preferred the curved drawings over chance level in the 2AFC task. These findings support the curvature effect using drawings of common-use objects (Corradi \& Munar, 2020). They also support the preference for curvature as a consistent effect 
558 between different experimental designs (Palumbo \& Bertamini, 2016; Chuquichambi et al.,

559

560

561

562

563

564

565

566

567

568

569

570

571

572

573

574

575

576

577

578

579

580

581

582

583

584

585

586

587

588

589

590

591

592

593

594

595

596

597

2021). Conversely, our findings diverge from those of some previous studies using images of real-objects. Munar et al. (2015) did not find preference for curved objects in a 2AFC task in the until-response condition. Similarly, using the same task and stimuli than Munar et al. (2015), Corradi et al. (2018) found that the effect of preference for curvature decreased as the presentation time increased. They suggested a higher influence of the meaning and contentrelated information of stimuli as the presentation time increased. In this regard, they found that the effect of preference for curvature was stronger when presenting abstract patterns in longer presentation time compared to brief presentations. With Japanese participants, Maezawa, Tanda and Kawahara (2020) did not find a preference for curvature using similar stimuli as Corradi et al. (2018) and like/dislike and rating scale tasks. A possible explanation of these divergences may be related to the interaction between the meaningful and representational content of the object, familiarity with its shape, and the artistic view of the drawings because of their design and artistic nature (Schroll, Schnurr \& Grewal, 2018).

The curved drawings were mostly preferred when the curved shape was selected as the most familiar or when the two shapes were selected as equally familiar, but not when the sharp-angled shape was selected as the most familiar. Further, in both experiments, preference for the curved drawings was higher when the curved shape was selected as the most familiar than when both shapes were selected as equally familiar. These findings support familiarity as a strong predictor of preference (Reber, Winkielman \& Schwarz, 1998; Reber, Schwarz \& Winkielman, 2004; Verhaeghen, 2018; Chmiel \& Schubert, 2019). However, they also suggest that familiarity is not the only factor determining preference for curvature because participants still preferred the curved drawings over the sharp-angled ones when the two shapes of the objects were selected as equally familiar. Moreover, there was no preference for the sharp-angled drawings when the sharp-angled shape was selected as the most familiar. In addition, these findings might support curvature as one of the diverse fluency-enhancing variables that explains the relationship between prototypicality and attractiveness (Winkielman et al., 2006).

Our results on the relationship between preference for curvature and familiarity might also be connected to the predominant role of curvature on shape's perception (Pasupathy \& Connor, 2002). Our visual system might integrate curved features more efficiently because they tend to match the statistic regularities of the natural environment (Sigman et al., 2001; Bertamini, Palumbo \& Redies, 2019; Stanischewski et al., 2020). Relatedly, our results might also be explained because of a higher frequency of curved contours in natural scenes (Ruta et al., 2019). In a recent study, Yue, Robert and Ungerleider (2020) found a specialized cortical network for curvature processing in humans. They suggested the interaction between preference for curvilinearity with central-peripheral processing biases as an important organizing principle for temporal cortex topography. Interestingly, they also found a possible link between curvaturepreferring areas and face-selective areas. This study also dealt with curvature as a metric property. However, Amir, Biederman and Hayworth $(2011,2012)$ showed greater sensitivity to the non-accidental property related to the difference between curved and straight contours than to

Peer) reviewing PDF | (2021:01:57478:2:0:NEW 15 Jun 2021) 
598 the metric property of curvature. Our brain might represent the non-accidental property in a 599 different way than the metric property of curvature. Altogether, these studies and the interaction 600 between the representational nature of the objects and the artistic characteristics of the drawings 601 within the same stimuli may explain our results of the role of familiarity in preference for 602 curvature.

603 However, the current research line on preference for curvature leaves open the role that could 604 play the phenomenology of how space appears to the perceiver. In particular, some artists use a 605 curvilinear perspective, instead of a linear perspective because it is closer to the viewer's 606 experience that straight lines in nature can be perceived as curved ones(Pepperell, 2012;

607 Pepperell \& Haertel, 2014). On the other hand, a curved line can even appear as a straight line

608

609

610

611

612

613

614

615

616

617

618

619

620

621

622

623

624

625

626

627

628

629

630

631

632

633

634

635

636

637 when viewed head-on, or circles in the peripheral visual field can appear polygonal in shape (Baldwin et al., 2016). Further research is needed to address this issue.

Besides the role of object characteristics, previous studies reported that individual measures also can modulate preference for curvature (e.g., Cotter et al., 2017; Silvia \& Barona, 2009). In Experiment 1, we only found that higher scores in the HB subscale predicted higher preference for all the drawings. However, we found some individual differences in Experiment 2, which leads us to suggest that the $2 \mathrm{AFC}$ task is more sensitive to finding them than the liking rating task. Specifically, participants with higher scores in the HB and A subscales showed a higher preference for curvature. The influence of the HB type of intuition in preference for curvature might be explained because curved contours facilitate fluent global processing of the stimuli (Reber, Schwarz \& Winkielman, 2004; Gómez-Puerto, Munar \& Nadal, 2016). On the other hand, the relationship between the A subscale and the preference for curvature could result from associations with positive valence underlying preference for curvature (Palumbo, Ruta \& Bertamini, 2015).

Our results also showed that higher scores in the Unconventionality facet predicted less preference for the curved drawings in Experiment 2. This might be related to the idea that the sharp-angled shapes are perceived as more avant-garde (Ruta et al., 2021) and unconventional people tend to show a higher preference for innovative designs. Interestingly, Cotter et al. (2017) found that higher unconventionality scores predicted more preference for curvature using irregular polygons. However, they found no effect using arrays of circles and hexagons. Using the same arrays of circles and hexagons, Silvia and Barona (2009) found preference for curvature in participants without art training - probably more conventional people- but there was no effect with art-trained participants - probably more unconventional people. Artists may show more unconventional thinking and express it in their art because this may make their work more impactful than more conventional artistic styles (Stamkou, van Kleef \& Homan, 2018). Conversely, these authors found preference for curvature in art-trained participants but not in participants without training when they rated complex polygons. Considering these studies, preference for curvature might be higher in art-trained and unconventional participants when the stimuli are more complex. However, we found no influence on preference for curvature from the Art interest and Art knowledge Scales, as in Corradi et al. (2019b). These authors reported that 
638 the influence of art interest and art knowledge on aesthetic sensitivity was inconsistent.

639 Altogether, our findings suggest that the influence of individual differences in preference for

640 curvature might depend on the kind of stimuli.

641 On the other hand, we found significant positive correlations between the results of both

642 experiments. The difference in liking ratings between curved and sharp-angled drawings

643 (Experiment 1), and the preference choices for the curved drawings (Experiment 2) showed a

644 similar pattern of preference. This finding supports a consistent and predictable preference for

645 drawings as representational images (Vessel \& Rubin, 2010; Schepman et al., 2015; Schepman,

646 Rodway \& Pullen, 2015). Although drawings also have art-related characteristics, our results

647 indicate that these characteristics did not weaken the preference consistency between

648 participants. On the other hand, the highly positive correlation between the familiarity selection

649 tasks endorse a robust concept of familiarity of object drawings regardless of the participants.

650 A possible limitation of this study is that we used a subjective measure of familiarity. The

651 familiarity values came from the direct response of the shape participants considered familiar for

652 the objects. Previous studies used measures based on the exposure time or the number of

653 presentations of the stimulus, that is, a process of familiarization (e.g., Berlyne, 1970, 1971;

654 Tinio \& Leder, 2009). However, Sluckin, Hargreaves and Colman (1982) argued that subjective

655 measures of familiarity, compared to objective measures, might be more suitable because of a

656 larger variance within each individual and stimulus. Moreover, the drawings involved content-

657 related information. Repeated exposure would likely lead to habituation and, as a consequence,

658 preference could decline (Biederman \& Vessel, 2006). Using subjective measures, participants

659 only need a single presentation of the stimulus to evaluate its representational content as more or

660 less familiar. Moreover, the subjective familiarity of the shape of an object might be modulated

661 by participant's individual differences. Thus, future studies could assess the role of individual

662 differences on shape familiarity in order to complement our findings of the relationship between

663 these variables with preference for curvature.

\section{Conclusions}

665 In summary, we found preference for curvature using drawings of common-use objects in two 666 experiments. The curved shapes of the objects were also selected as the most familiar ones in

667 both experiments. When the curved shapes were selected as the most familiar, and when both

668 shapes were selected as equally familiar, participants showed preference for the curved drawings.

669 However, when the sharp-angled shapes were selected as the most familiar, participants did not

670 show preference for curvature or for angularity. These findings support the idea that shape

671 familiarity modulates preference for drawings of common-use objects. However, they also

672 indicate that the influence of familiarity is not the only factor explaining the preference for

673 curved drawings. The influence of individual differences in preference for the drawings

674 suggested that the kind of stimuli and the experimental task may predict divergencies across

675 studies and measures. Correlation analyses between experiments also supported a consistent

676 relationship of preference between tasks, and a coherent concept of familiarity of the same pair 
677 of object drawings. Altogether, our findings endorse the curvature effect using drawings of

678 common-use objects and familiarity as an important predictor of preference.

679

680

\section{Acknowledgements}

681

682

We are grateful to Marco Bertamini, and Michele Sinico for making the stimuli set available.

\section{References}

683

684

Amir O, Biederman I, Hayworth KJ. 2011. The neural basis for shape preferences. Vision Research 51:2198-2206. DOI: 10.1016/j.visres.2011.08.015.

685

686

687

688

689

690

691

692

693

694

695

696

697

698

699

700

701

702

703

704

705

706

707

708

709

710

711

712

713

714

715

716

717

718

719

720

721

Amir O, Biederman I, Hayworth KJ. 2012. Sensitivity to nonaccidental properties across various shape dimensions. Vision Research 62:35-43. DOI: 10.1016/j.visres.2012.03.020.

Baayen RH, Davidson DJ, Bates DM. 2008. Mixed-effects modeling with crossed random effects for subjects and items. Journal of Memory and Language 59:390-412. DOI: 10.1016/j.jml.2007.12.005.

Baldwin J, Burleigh A, Pepperell R, Ruta N. 2016. The Perceived Size and Shape of Objects in Peripheral Vision. i-Perception 7:204166951666190. DOI: 10.1177/2041669516661900.

Bamford S, Broyd SJ, Benikos N, Ward R, Wiersema JR, Sonuga-Barke E. 2015. The late positive potential: A neural marker of the regulation of emotion-based approach-avoidance actions? Biological Psychology 105:115-123. DOI: 10.1016/J.BIOPSYCHO.2015.01.009.

Bar M, Neta M. 2006. Humans prefer curved visual objects. Psychological Science 17:645-648. DOI: $10.1111 /$ j.1467-9280.2006.01759.x.

Bar M, Neta M. 2007. Visual elements of subjective preference modulate amygdala activation. Neuropsychologia 45:2191-2200. DOI: 10.1016/j.neuropsychologia.2007.03.008.

Barr DJ, Levy R, Scheepers C, Tily HJ. 2013. Random effects structure for confirmatory hypothesis testing: Keep it maximal. Journal of Memory and Language 68:255-278. DOI: 10.1016/j.jml.2012.11.001.

Bates D, Mächler M, Bolker B, Walker S. 2015. Fitting Linear Mixed-Effects Models using lme4. Journal of Statistical Software 67:1-48. DOI: 0.18637/jss.v067.i01.

Berlyne DE. 1970. Novelty, complexity, and hedonic value. Perception \& Psychophysics 8:279286. DOI: 10.3758/BF03212593.

Berlyne DE. 1971. Aesthetics and Psychobiology. New York: Appleton-Century-Crofts.

Bertamini M, Palumbo L, Gheorghes TN, Galatsidas M. 2016. Do observers like curvature or do they dislike angularity? British Journal of Psychology 107:154-178. DOI: 10.1111/bjop.12132.

Bertamini M, Palumbo L, Redies C. 2019. An Advantage for Smooth Compared With Angular Contours in the Speed of Processing Shape. Journal of Experimental Psychology: Human Perception and Performance 45:1304-1318. DOI: 10.1037/xhp0000669.

Bertamini M, Rampone G, Makin ADJ, Jessop A. 2019. Symmetry preference in shapes, faces, flowers and landscapes. PeerJ 2019:e7078. DOI: 10.7717/peerj.7078.

Bertamini M, Sinico M. 2019. A Study of Objects With Smooth or Sharp Features Created as Line Drawings by Individuals Trained in Design. Empirical Studies of the Arts 39:61-77. DOI: $10.1177 / 0276237419897048$.

Biederman I, Vessel EA. 2006. Perceptual pleasure and the brain. American Scientist 94:249255.

Bornstein RF. 1989. Exposure and Affect: Overview and Meta-Analysis of Research, 19681987. Psychological Bulletin 106:265-289. DOI: 10.1037/0033-2909.106.2.265.

Peer] reviewing PDF | (2021:01:57478:2:0:NEW 15 Jun 2021) 
722

723

724

725

726

727

728

729

730

731

732

733

734

735

736

737

738

739

740

741

742

743

744

745

746

747

748

749

750

751

752

753

754

755

756

757

758

759

760

761

762

763

764

765

766

767

Brauer M, Curtin JJ. 2018. Linear mixed-effects models and the analysis of nonindependent data: A unified framework to analyze categorical and continuous independent variables that vary within-subjects and/or within-items. Psychological Methods 23:389-411. DOI: 10.1037/met0000159.

Chatterjee A, Widick P, Sternschein R, Smith WB, Bromberger B. 2010. The Assessment of Art Attributes. Empirical Studies of the Arts 28:207-222. DOI: 10.2190/EM.28.2.f.

Child IL. 1962. Personal preferences as an expression of aesthetic sensitivity. Journal of Personality 30:496-512. DOI: 10.1111/j.1467-6494.1962.tb02319.x.

Child IL. 1965. Personality correlates of esthetic judgment in college students. Journal of Personality 33:476-511. DOI: 10.1111/j.1467-6494.1965.tb01399.x.

Chmiel A, Schubert E. 2019. Unusualness as a predictor of music preference. Musicae Scientiae 23:426-441. DOI: 10.1177/1029864917752545.

Chuquichambi EG, Corradi GB, Munar E, Rosselló-Mir J. 2021. When Symmetric and Curved Visual Contour Meet Intentional Instructions: Hedonic Value and Preference. Quarterly Journal of Experimental Psychology. DOI: 10.1177/17470218211021593.

Chuquichambi EG, Rey C, Llames R, Escudero JT, Dorado A, Munar E. 2020. Circles Are Detected Faster Than Downward-Pointing Triangles in a Speeded Response Task. Perception 49:1026-1042. DOI: 10.1177/0301006620957472.

Corradi G, Belman M, Currò T, Chuquichambi EG, Rey C, Nadal M. 2019a. Aesthetic sensitivity to curvature in real objects and abstract designs. Acta Psychologica 197:124130. DOI: 10.1016/j.actpsy.2019.05.012.

Corradi G, Chuquichambi EG, Barrada JR, Clemente A, Nadal M. 2019b. A new conception of visual aesthetic sensitivity. British Journal of Psychology 111: 630-658. DOI: 10.1111/bjop. 12427.

Corradi G, Munar E. 2020. The Curvature Effect. In: Nadal Roberts M, Vartanian O eds. The Oxford Handbook of Empirical Aesthetics. Oxford Univerisity Press, 35-52. DOI: 10.1093/oxfordhb/9780198824350.013.24.

Corradi G, Rosselló-Mir J, Vañó J, Chuquichambi E, Bertamini M, Munar E. 2018. The effects of presentation time on preference for curvature of real objects and meaningless novel patterns. British Journal of Psychology, 110: 670-685. DOI: 10.1111/bjop.12367.

Cotter KN, Silvia PJ, Bertamini M, Palumbo L, Vartanian O. 2017. Curve appeal: Exploring individual differences in preference for curved versus angular objects. $i$-Perception 8:1-17. DOI: $10.1177 / 2041669517693023$.

Davidson R, MacKinnon JG. 2000. Bootstrap tests: How many bootstraps? Econometric Reviews 19:55-68. DOI: 10.1080/07474930008800459.

Dazkir SS, Read MA. 2012. Furniture form and their influence on our emotional responses toward interior environments. Environment and Behavior 44:722-732. DOI: $10.1177 / 0013916511402063$.

Forsythe A, Mulhern G, Sawey M. 2008. Confounds in pictorial sets: The role of complexity and familiarity in basic-level picture processing. Behavior Research Methods 40:116-129. DOI: 10.3758/BRM.40.1.116.

Gómez-Puerto G, Munar E, Nadal M. 2016. Preference for Curvature: A Historical and Conceptual Framework. Frontiers in human neuroscience 9:712. DOI: 10.3389/fnhum.2015.00712.

Gross MD, Ervin SM, Anderson JA, Fleisher A. 1988. Constraints: Knowledge representation in design. Design Studies 9:133-143. DOI: 10.1016/0142-694X(88)90042-7.

PeerJ reviewing PDF | (2021:01:57478:2:0:NEW 15 Jun 2021) 
768

769

770

771

772

773

774

775

776

777

778

779

780

781

782

783

784

785

786

787

788

789

790

791

792

793

794

795

796

797

798

799

800

801

802

803

804

805

806

807

808

809

810

811

812

813

Hekkert P, Snelders HMJJ (Dirk). 1995. Prototypicality as an Explanatory Concept in Aesthetics: A Reply to Boselie (1991). Empirical Studies of the Arts 13:149-160. DOI: 10.2190/kyra-r5ur-ara8-cxfn.

Hekkert P, Snelders D, Wieringen PCW. 2003. 'Most advanced, yet acceptable': Typicality and novelty as joint predictors of aesthetic preference in industrial design. British Journal of Psychology 94:111-124. DOI: 10.1348/000712603762842147.

Hoffman DD, Singh M. 1997. Salience of visual parts. Cognition 63:29-78. DOI: 10.1016/S0010-0277(96)00791-3.

Hox JJ. 2010. Multilevel analysis. Techniques and applications. New York: Routeledge.

Hůla M, Flegr J. 2016. What flowers do we like? The influence of shape and color on the rating of flower beauty. PeerJ 2016:e2106. DOI: 10.7717/peerj.2106.

Kavakli M, Suwa M, Gero J, Purcell T. 1999. Sketching Interpretation in Novice and Experts Designers. In: J. S. Gero \& B. Tversky ed. Visual and spatial reasoning in design. Cambridge, MA: Key Centre of Design Computing and Cognition, University of Sydney, 209-220.

Koenderink JJ. 1984. What Does the Occluding Contour Tell Us about Solid Shape? Perception 13:321-330. DOI: $10.1068 / \mathrm{p} 130321$.

Kozbelt A, Seidel A, ElBassiouny A, Mark Y, Owen DR. 2010. Visual selection contributes to artists' advantages in realistic drawing. Psychology of Aesthetics, Creativity, and the Arts 4:93-102. DOI: 10.1037/a0017657.

Lawson B. 1980. How designers think. Amsterdam, the Netherlands: Elsevier.

Leder H. 2001. Determinants of Preference: When do we like What we Know? Empirical Studies of the Arts 19:201-211. DOI: 10.2190/5tae-e5cv-xjal-3885.

Leder H, Belke B, Oeberst A, Augustin D. 2004. A model of aesthetic appreciation and aesthetic judgments. British Journal of Psychology 95:489-508. DOI: 10.1348/0007126042369811.

Leder H, Carbon C-C. 2005. Dimensions in appreciation of car interior design. Applied Cognitive Psychology 19:603-618. DOI: 10.1002/acp.1088.

Leder H, Tinio PPL, Brieber D, Kröner T, Jacobsen T, Rosenberg R. 2019. Symmetry Is Not a Universal Law of Beauty. Empirical Studies of the Arts 37:104-114. DOI: $10.1177 / 0276237418777941$.

Lee K, Ashton MC. 2004. Psychometric properties of the HEXACO personality inventory. Multivariate Behavioral Research 39:329-358. DOI: 10.1207/s15327906mbr3902_8.

Lenth R V. 2016. Least-Squares Means: The R Package 1smeans. Journal of Statistical Software 69. DOI: 10.18637 /jss.v069.i01.

Maezawa T, Tanda T, Kawahara JI. 2020. Replicability of the Curvature Effect as a Function of Presentation Time and Response Measure in Japanese Observers. i-Perception 11:204166952091520. DOI: 10.1177/2041669520915204.

Mathôt S, Schreij D, Theeuwes J. 2012. OpenSesame: An open-source, graphical experiment builder for the social sciences. Behavior Research Methods 44:314-324. DOI: 10.3758/s13428-011-0168-7.

Matindale C. 1990. The clockwork muse: The predictability of artistic change. - PsycNET. Basic Books.

McCrae RR, Costa PT. 2004. A contemplated revision of the NEO Five-Factor Inventory. Personality and Individual Differences 36:587-596. DOI: 10.1016/S0191-8869(03)001181.

Munar E, Gómez-Puerto G, Call J, Nadal M. 2015. Common Visual Preference for Curved 
814 Contours in Humans and Great Apes. PLoS ONE 10:e0141106. DOI:

$815 \quad$ 10.1371/journal.pone.0141106.

816 Naghibi Rad P, Shahroudi AA, Shabani H, Ajami S, Lashgari R. 2019. Encoding Pleasant and

817 Unpleasant Expression of the Architectural Window Shapes: An ERP Study. Frontiers in

818 Behavioral Neuroscience 13. DOI: 10.3389/fnbeh.2019.00186.

819

820

821

822

823

824

825

826

827

828

829

830

831

832

833

834

835

836

837

838

839

840

841

842

843

844

845

846

847

848

849

850

851

852

853

854

855

856

857

858

859

Van Oel CJ, Van den Berkhof FWD. 2013. Consumer preferences in the design of airport passenger areas. Journal of Environmental Psychology 36:280-290. DOI: 10.1016/j.jenvp.2013.08.005.

Palumbo L, Bertamini M. 2016. The Curvature Effect: A Comparison Between Preference Tasks. Empirical Studies of the Arts 34:35-52. DOI: 10.1177/0276237415621185.

Palumbo L, Ogden R, Makin ADJ, Bertamini M. 2014. Examining visual complexity and its influence on perceived duration. Journal of Vision 14:3-3. DOI: 10.1167/14.14.3.

Palumbo L, Rampone G, Bertamini M, Sinico M, Clarke E, Vartanian O. 2020. Visual preference for abstract curvature and for interior spaces: Beyond undergraduate student samples. Psychology of Aesthetics, Creativity, and the Arts. DOI: 10.1037/aca0000359.

Palumbo L, Ruta N, Bertamini M. 2015. Comparing angular and curved shapes in terms of implicit associations and approach/avoidance responses. Plos One 10:e0140043. DOI: 10.1371/journal.pone.0140043.

Park J, Shimojo E, Shimojo S. 2010. Roles of familiarity and novelty in visual preference judgments are segregated across object categories. Proceedings of the National Academy of Sciences 107:14552-14555. DOI: 10.1073/pnas.1004374107.

Pasupathy A, Connor CE. 2002. Population coding of shape in area V4. Nature Neuroscience 5:1332-1338. DOI: 10.1038/nn972.

Penacchio O, Otazu X, Dempere-Marco L. 2013. A Neurodynamical Model of Brightness Induction in V1. PLoS ONE 8:64086. DOI: 10.1371/journal.pone.0064086.

Penacchio O, Wilkins AJ. 2015. Visual discomfort and the spatial distribution of Fourier energy. Vision Research 108:1-7. DOI: 10.1016/j.visres.2014.12.013.

Pepperell R. 2012. The perception of art and the science of perception. Human Vision and Electronic Imaging XVII 8291:829113. DOI: 10.1117/12.914774.

Pepperell R, Haertel M. 2014. Do artists use linear perspective to depict visual space? Perception 43:395-416. DOI: 10.1068/p7692.

Pretz JE, Brookings JB, Carlson LA, Humbert TK, Roy M, Jones M, Memmert D. 2014. Development and Validation of a New Measure of Intuition: The Types of Intuition Scale. Journal of Behavioral Decision Making 27:454-467. DOI: 10.1002/bdm.1820.

Purcell AT, Gero JS. 1998. Drawings and the design process. Design Studies 19:389-430. DOI: 10.1016/s0142-694x(98)00015-5.

R Core Team (2018). R: The R Project for statistical computing. A language and environment for statistical computing. Vienna, Austria: R Foundation for Statistical Computing. Version 3.5.0. URL. https://www.r-project.org/

Reber R, Schwarz N, Winkielman P. 2004. Affect and processing dynamics. Personality and social psychology review : an official journal of the Society for Personality and Social Psychology, Inc 8:364-82. DOI: 10.1207/s15327957pspr0804_3.

Reber R, Winkielman P, Schwarz N. 1998. Effects of Perceptual Fluency on Affective Judgments. Psychological Science 9:45-48. DOI: 10.1111/1467-9280.00008.

Reber R, Wurtz P, Zimmermann TD. 2004. Exploring "fringe" consciousness: The subjective experience of perceptual fluency and its objective bases. Consciousness and cognition

PeerJ reviewing PDF | (2021:01:57478:2:0:NEW 15 Jun 2021) 
860

861

862

863

864

865

866

867

868

869

870

871

872

873

874

875

876

877

878

879

880

881

882

883

884

885

886

887

888

889

890

891

892

893

894

895

896

897

898

899

900

901

902

903

904

905

13:47-60. DOI: 10.1016/S1053-8100(03)00049-7.

Rodway P, Kirkham J, Schepman A, Lambert J, Locke A. 2016. The Development of Shared Liking of Representational but not Abstract Art in Primary School Children and Their Justifications for Liking. Frontiers in Human Neuroscience 10:21. DOI: 10.3389/fnhum.2016.00021.

Ruta N, Mastandrea S, Penacchio O, Lamaddalena S, Bove G. 2019. A comparison between preference judgments of curvature and sharpness in architectural façades. Architectural Science Review 62:171-181. DOI: 10.1080/00038628.2018.1558393.

Ruta N, Vañó J, Pepperell R, Corradi GB, Chuquichambi EG, Rey C, Munar E. 2021. Preference for paintings is also affected by curvature. Psychology of Aesthetics, Creativity, and the Arts. DOI: 10.1037/aca0000395.

Schepman A, Rodway P, Pullen SJ. 2015. Greater cross-viewer similarity of semantic associations for representational than for abstract artworks. Journal of Vision 15:12-12. DOI: $10.1167 / 15.14 .12$.

Schepman A, Rodway P, Pullen SJ, Kirkham J. 2015. Shared liking and association valence for representational art but not abstract art. Journal of Vision 15:1-10. DOI: 10.1167/15.5.11.

Schroll R, Schnurr B, Grewal D. 2018. Humanizing Products with Handwritten Typefaces. Journal of Consumer Research 45:648-672. DOI: 10.1093/jcr/ucy014.

Sigman M, Cecchi GA, Gilbert C. D, Magnasco MO. 2001. On a common circle: Natural scenes and Gestalt rules. Proceedings of the National Academy of Sciences 98:1935-1940. DOI: 10.1073/pnas.031571498.

Silvia PJ, Barona CM. 2009. Do People Prefer Curved Objects? Angularity, Expertise, and Aesthetic Preference. Empirical Studies of the Arts 27:25-42. DOI: 10.2190/EM.27.1.b.

Singmann H, Bolker B, Westfall J, Aust F. 2016. afex: Analysis of factorial experiments. R Package Version 0.18-0.

Sluckin W, Hargreaves DJ, Colman AM. 1982. Some experimental studies of familiarity and liking*. Bulletin of The British Psychological Society 35:189-194.

Snijders TAB, Bosker RJ. 2012. Multilevel analysis. An introduction to basic and advanced multilevel modeling. London: Sage Publications.

Stamkou E, van Kleef GA, Homan AC. 2018. The art of influence: When and why deviant artists gain impact. Journal of Personality and Social Psychology 115:276-303. DOI: $10.1037 /$ pspi0000131.

Stanischewski S, Altmann CS, Brachmann A, Redies C. 2020. Aesthetic Perception of Line Patterns: Effect of Edge-Orientation Entropy and Curvilinear Shape. i-Perception 11. DOI: $10.1177 / 2041669520950749$.

Tinio PPL, Leder H. 2009. Just how stable are stable aesthetic features? Symmetry, complexity, and the jaws of massive familiarization. Acta Psychologica 130:241-250. DOI: 10.1016/j.actpsy.2009.01.001.

Vartanian O, Navarrete G, Chatterjee A, Fich LB, Leder H, Modroño C, Nadal M, Rostrup N, Skov M. 2013. Impact of contour on aesthetic judgments and approach-avoidance decisions in architecture. Proceedings of the National Academy of Sciences of the United States of America 110:10446-53. DOI: 10.1073/pnas.1301227110.

Vartanian O, Navarrete G, Chatterjee A, Fich LB, Leder H, Rostrup N, Skov M, Corradi G, Nadal M, Rostrup N, Skov M. 2017. Preference for curvilinear contour in interior architectural spaces: Evidence from experts and nonexperts preference. Psychology of Aesthetics , Creativity, and the Arts 13:110-116. DOI: 10.1037/aca0000150.

Peer) reviewing PDF | (2021:01:57478:2:0:NEW 15 Jun 2021) 
906

907

908

909

910

911

912

913

914

915

916

917

918

919

920

921

922

923

924

925

926

927

928

929
Verhaeghen P. 2018. Once More, with Feeling: the Role of Familiarity in the Aesthetic Response. Psychological Record 68:379-384. DOI: 10.1007/s40732-018-0312-1.

Vessel EA, Rubin N. 2010. Beauty and the beholder: Highly individual taste for abstract, but not real-world images. Journal of Vision 10:1-14. DOI: 10.1167/10.2.18.

Wang L, Yu Y, Li O. 2019. The typeface curvature effect: The role of typeface curvature in increasing preference toward hedonic products. Psychology \& Marketing 37:1118-1137. DOI: $10.1002 /$ mar.21287.

Westerman S, Gardner PH, Sutherland EJ, White T, Jordan K, Watts D, Wells S. 2012. Product design: Preference for rounded versus angular design elements. Psychology \& Marketing 29:595-605. DOI: 10.1002/mar.

Whitfield TWA. 1983. Predicting preference for familiar, everyday objects: An experimental confrontation between two theories of aesthetic behaviour. Journal of Environmental Psychology 3:221-237. DOI: 10.1016/S0272-4944(83)80002-4.

Whitfield TWA, Slatter PE. 1979. The effects of categorization and prototypicality on aesthetic choice in a furniture selection task. British Journal of Psychology 70:65-75. DOI: 10.1111/j.2044-8295.1979.tb02144.x.

Winkielman P, Halberstadt J, Fazendeiro T, Catty S. 2006. Prototypes are attractive because they are easy on the mind. Psychological Science 17:799-806. DOI: 10.1111/j.14679280.2006.01785.x.

Yue X, Robert S, Ungerleider LG. 2020. Curvature processing in human visual cortical areas. NeuroImage 222:117295. DOI: 10.1016/j.neuroimage.2020.117295.

Zajonc R. 1968. Attitudinal effects of mere exposure. Journal of Personality and Social Psychology 9:1-27. DOI: 10.1037/h0025848. 
Figure 1

Examples of the pairs of drawings (IUAV image database).

Each pair has a curved and sharp-angled version. Left-side, computer-made. Right-side, hand-made. Top, not shaded. Bottom, shaded.

Computer-made
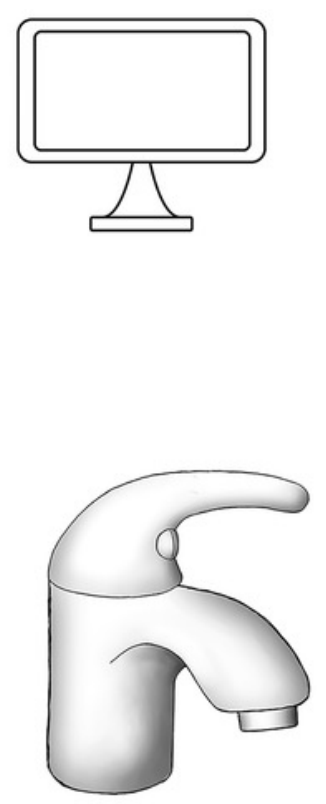

Curved
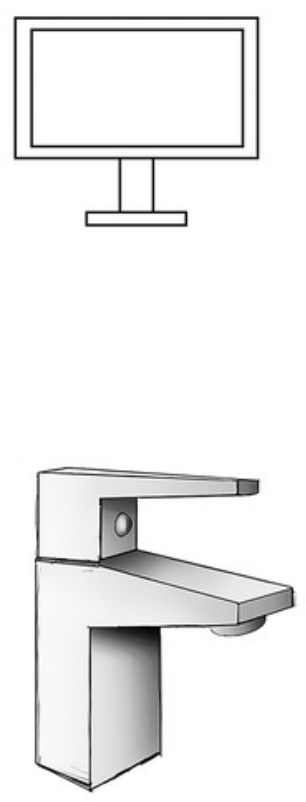

Sharp-angled

Hand-made
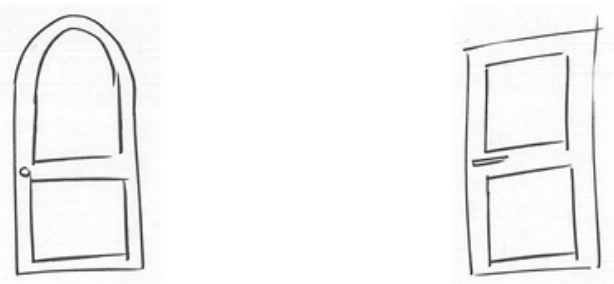

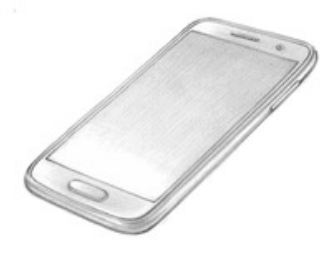

Curved

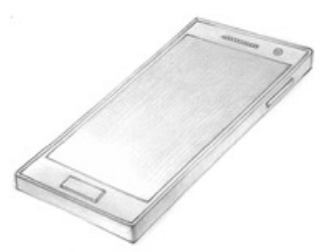

Sharp-angled

Contour 


\section{Figure 2}

Trials sequence in the preference tasks of experiments 1 and 2.

(A) An example trial in the liking rating task from Experiment 1. (B) An example trial in the two-alternative forced-choice task from Experiment 2. The example shows that the left object was selected. In the next slide, the left object (selected) and the right object (non-selected) simulated approach and avoidance actions, respectively.

A

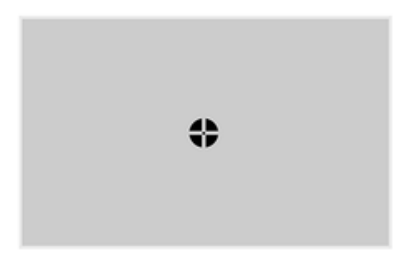

$500 \mathrm{~ms}$

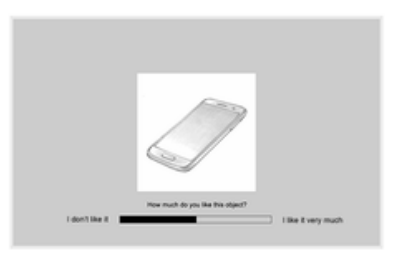

Until response

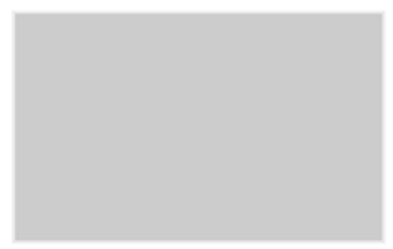

$1000-1500 \mathrm{~ms}$

B

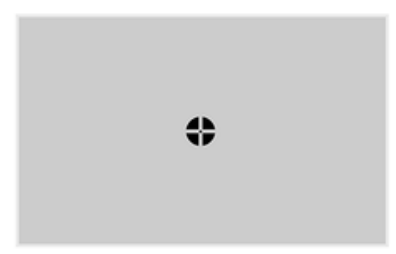

$500 \mathrm{~ms}$

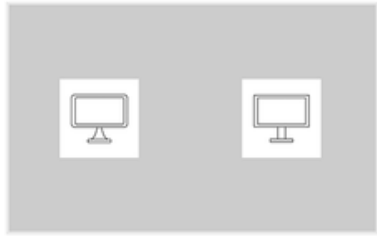

Select one (Until response)

$\leftarrow \rightarrow$

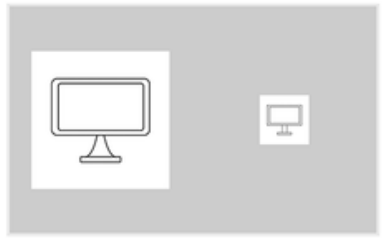

Approach left | Avoidance right $1000 \mathrm{~ms}$

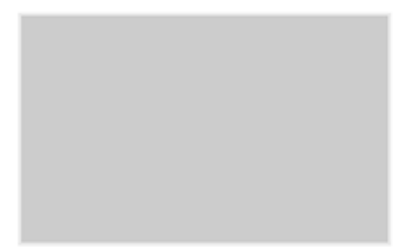

$1000-1500 \mathrm{~ms}$ 


\section{Figure 3}

Liking ratings and familiarity selections of Experiment 1.

(A) Mean liking ratings for the curved and sharp-angled drawings. (B) Mean liking ratings for the drawings within the three alternative responses of the Familiarity selection task. Left graphic represents familiarity selections for the curved shapes, middle graphic represents both shapes selected as equally familiar, and right graphic represents familiarity selections for the sharp-angled shapes. Each one of these graphics show mean liking ratings for the curved and sharp-angled drawings. The curved drawings were liked more when the curved shapes were selected as the most familiar ones, or when both shapes were selected as equally familiar, but not when the sharp-angled shapes were selected as the most familiar ones. Error bars represent $95 \% \mathrm{Cl}(* * p \leq .01, * * * p \leq .001$, n.s.: not significant).
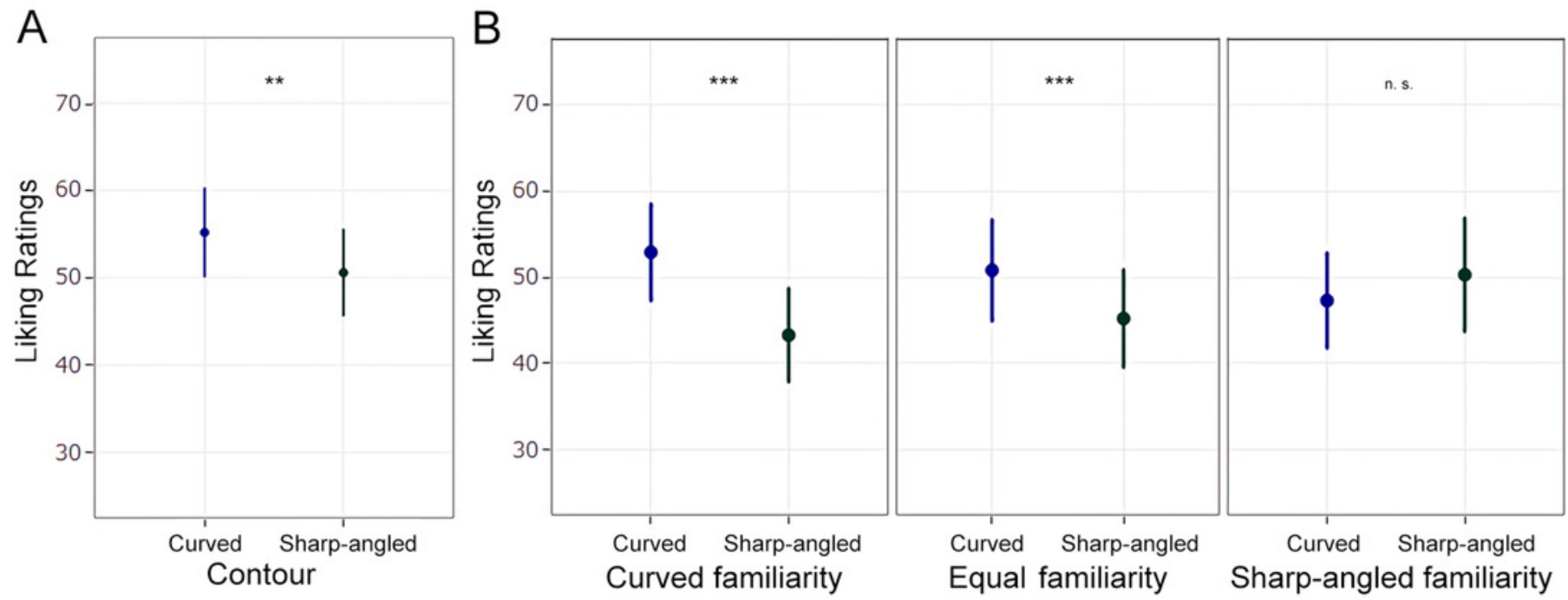
Figure 4

Preference choices and familiarity selections of Experiment 2.

(A) Mean choices of the curved and sharp-angled drawings in the 2AFC task. (B) Probability of choosing the curved drawings in the 2AFC task within the three alternative responses of the Familiarity selection task. Familiarity selections for the curved shapes and both shapes selected as equally familiar predicted a higher probability of choosing the curved drawings in the 2AFC. Error bars represent $95 \% \mathrm{Cl}(* * * p<.001)$.
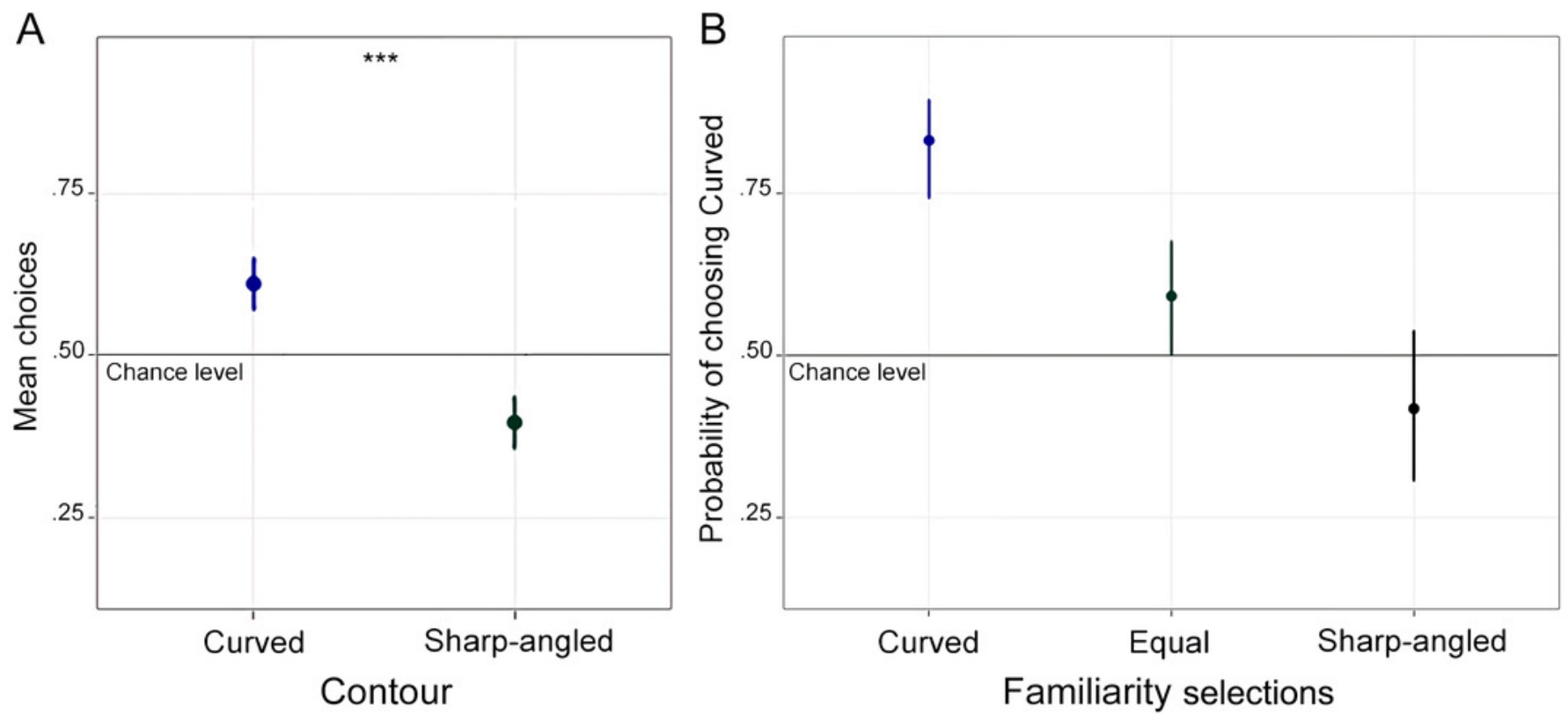
Figure 5

Scatterplots showing the relation between the data from experiments 1 and 2 .

(A) Relation between the liking ratings (Experiment 1) and the curved choices in the 2AFC (Experiment 2). (B) Relation between the familiarity selections data of Experiment 1 and 2. Each point represents a pair of drawings. All p's $<.001$.
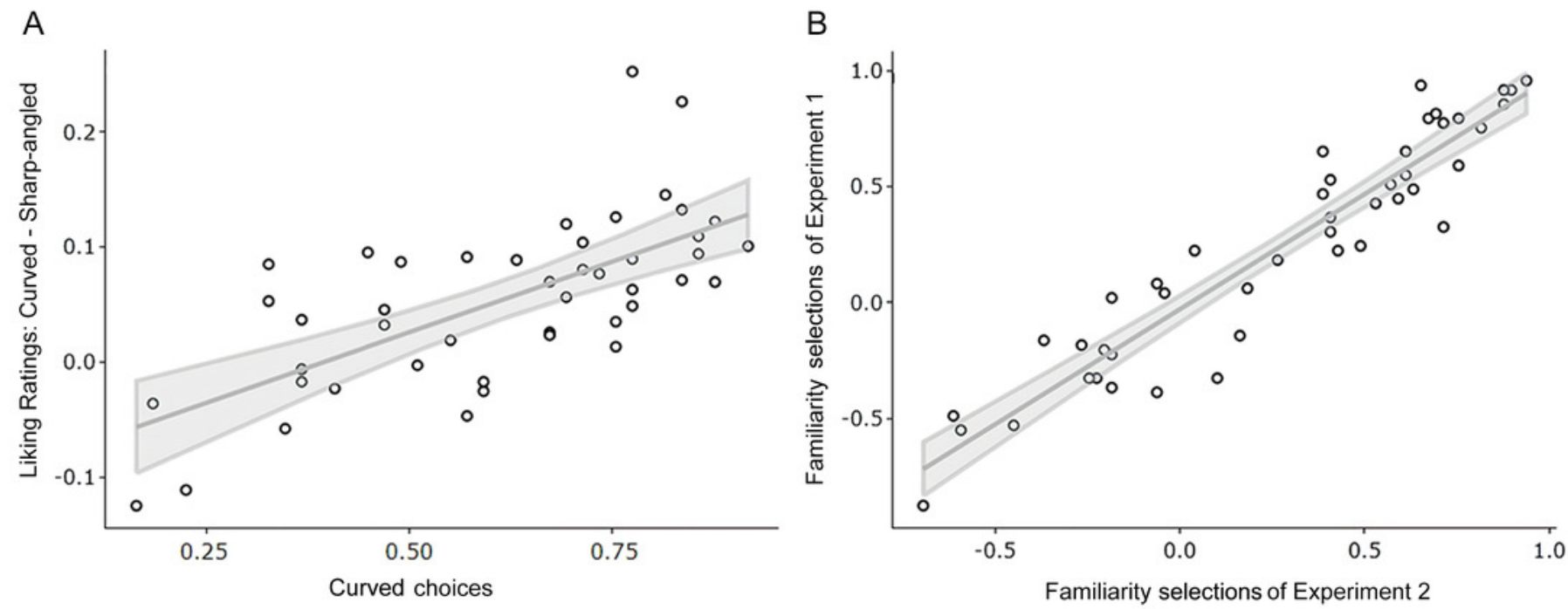


\section{Table $\mathbf{1}$ (on next page)}

Descriptive statistics for the individual differences measures of Experiment $1(n=49)$.

Score ranges: Art interest $(0-30)$, Art knowledge $(0-18)$, Openness to experience $(12-60)$, Unconventionality (4 - 20), HA (3 - 15), HB (4 - 20), I (8 - 40), A (8 - 40). 


\begin{tabular}{lcccc}
\hline Variable & Mean & Median & SD & Min - Max \\
\hline Art interest & 10.6 & 12 & 5.44 & $1-20$ \\
Art knowledge & 1.43 & 1 & 2.03 & $0-11$ \\
NEO: Openness to experience & 47.4 & 48 & 5.94 & $30-59$ \\
HEXACO: Unconventionality & 3.61 & 3.75 & .57 & $2.25-5$ \\
TIntS: Holistic Abstract (HA) & 8.4 & 8 & 2 & $3-14$ \\
TIntS: Holistic Big picture (HB) & 13.3 & 13 & 2.47 & $8-19$ \\
TIntS: Inferential (I) & 28.5 & 29 & 3.4 & $19-35$ \\
TIntS: Affective (A) & 25 & 25 & 5.03 & $16-36$ \\
\hline
\end{tabular}




\section{Table 2 (on next page)}

Descriptive statistics for the individual differences measures of Experiment $2(n=49)$.

Score ranges: Art interest $(0-30)$, Art knowledge $(0-18)$, Openness to experience $(12-60)$, Unconventionality (4 - 20), HA (3 - 15), HB (4 - 20), I (8 - 40), A (8 - 40). 


\begin{tabular}{lcccc}
\hline Variable & Mean & Median & SD & Min - Max \\
\hline Art interest & 10 & 9 & 6.22 & $0-26$ \\
Art knowledge & 2.35 & 1 & 2.94 & $0-12$ \\
NEO: Openness to experience & 46.3 & 45 & 5.53 & $36-58$ \\
HEXACO: Unconventionality & 3.58 & 3.5 & .58 & $2.5-5$ \\
TIntS: Holistic Abstract (HA) & 7.94 & 8 & 2.21 & $3-13$ \\
TIntS: Holistic Big picture (HB) & 12.8 & 12 & 2.55 & $7-20$ \\
TIntS: Inferential (I) & 29.5 & 30 & 3.33 & $20-38$ \\
TIntS: Affective (A) & 25.3 & 25 & 5.53 & $12-35$
\end{tabular}

1

2 